\title{
Evaluación de la consolidación de conocimientos de la anatomía radicular mediante el uso de realidad aumentada por alumnos del posgrado de Endodoncia de la UNITEC
}

Evaluation of the consolidation of knowledge of root anatomy using augmented reality by students of the Postgraduate Endodontics of UNITEC

Avaliação da consolidação do conhecimento da anatomia radicular por meio do uso da realidade aumentada por alunos do programa de pósgraduação em Endodontia da UNITEC

Carlos Fidelmar Andrade Mosqueda Universidad Tecnológica de México - UNITEC México - Campus Marina-Cuitláhuac,

México eecarlosandrade@gmail.com https://orcid.org/0000-0002-6764-7234

\section{Resumen}

Los esquemas educativos actuales cada vez se centran más en modelos basados en competencias y en el aprovechamiento de los recursos tecnológicos. Estos elementos propician que los roles de los alumnos, profesores y autoridades educativas vayan cambiando y adaptándose en beneficio de un mejor proceso de enseñanza y aprendizaje. Por ello, es importante investigar si los diseños instruccionales basados en los nuevos paradigmas educativos son funcionales en la enseñanza de las diversas asignaturas del área odontológica. Con la participación de 10 residentes del posgrado de Endodoncia de la Universidad Tecnológica de México, campus Cuitláhuac, se realizó un estudio cuasiexperimental, en donde se creó un rotafolio tipo libro acerca de la anatomía radicular. Por medio de investigación bibliográfica, los residentes recopilaron información e imágenes acerca del tema y posteriormente, con el uso de plastilina profesional, crearon modelos anatómicos 3D 


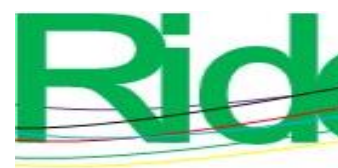

Revista Iberoamericana para la Investigación y el Desarrollo Educativo ISSN $2007-7467$

que se transformaron en imágenes digitales por medio de un software; a continuación, esas imágenes se utilizaron para diseñar y crear el rotafolio de realidad aumentada. Para evaluar la consolidación de conocimientos se utilizó una evaluación diagnóstica Knwoledge and Prior Study Inventory (KPSI) antes de la actividad, y una evaluación sumativa al terminar su implementación. La prueba diagnóstica mostró que $60 \%$ de los residentes iniciaron el posgrado con una categoría de conocimiento no consolidado. Después de implementar el diseño didáctico del rotafolio de realidad aumentada, creado por los alumnos con la guía y apoyo del docente, se observó que 90 \% de los residentes alcanzó en su evaluación la categoría de conocimiento consolidado; finalmente, se aplicó la prueba estadística de chi cuadrado $\left(\mathrm{X}^{2}\right)$ para determinar si existía relación entre las variables analizadas. El resultado de la prueba arrojó que la implementación del rotafolio de realidad aumentada impactaba en la consolidación del conocimiento de la anatomía radicular. En conclusión, los resultados obtenidos mostraron que incorporar los nuevos modelos educativos basados en competencias y nuevas tecnologías es favorable para la consolidación de conocimientos y aprendizajes instrumentales y procedimentales.

Palabras clave: aprendizajes, diseño didáctico, rotafolio, tecnología educativa, tratamiento de conductos.

\section{Abstract}

Current educational schemes increasingly focus on operational models in competencies and in the use of technological resources. These elements are conducive for the roles of students, teachers and educational authorities to change and adapt to benefit a better teaching-learning process. It is therefore important to investigate whether the instructional designs detected in the new educational paradigms are functional in teaching the various subjects in the dental area. With the participation of 10 residents of the Endodontic Postgraduate Course at the Technological University of Mexico Campus Marina-Cuitlahuac, a quasi-experimental study was carried out, a book-type Flipchart was created about root anatomy. Through bibliographic research the residents collected images and information about the topic, later with the use of professional clay they created 3D anatomical models that are transformed into digital images through software, then these images will be used to power and create the Flipchart of augmented reality. To evaluate the consolidation of knowledge a diagnostic evaluation (KPSI model) was used before the activity and a summative evaluation at the end 


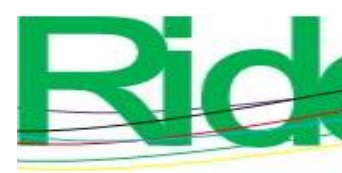

Revista Iberoamericana para la Investigación y el Desarrollo Educativo ISSN 2007 - 7467

of its implementation. The diagnostic test showed that $60 \%$ of the residents started postgraduate studies with an unconsolidated knowledge category. After implementing the didactic design of the augmented reality flip chart, created by the students with the guidance and support of the teacher, it was observed that $90 \%$ of the residents reached the category of consolidated knowledge in their evaluation. Finally, the Chi-square statistical test (X2) was applied to determine if there was a relationship between the variables analyzed, the result of the test showed that the implementation of the augmented reality flipchart had an impact on the consolidation of knowledge of root anatomy. In conclusion, the results obtained in this study showed that incorporates the new educational models, based in competencies and new technologies, in the teaching-learning process of dental anatomy is favorable for the consolidation of instrumental and procedural knowledge and learning.

Keywords: Learnings, Didactic design, Flipchart, Educative technology, Root canal treatment.

\section{Resumo}

Os esquemas educacionais atuais estão cada vez mais focados em modelos baseados em competências e no uso de recursos tecnológicos. Esses elementos permitem que os papéis dos alunos, professores e autoridades educacionais mudem e se adaptem para beneficiar um melhor processo de ensino e aprendizagem. Por esse motivo, é importante investigar se os desenhos instrucionais baseados nos novos paradigmas educacionais são funcionais no ensino das diversas disciplinas da área odontológica. Com a participação de 10 residentes do curso de pós-graduação em Endodontia da Universidade Tecnológica do México, campus Cuitláhuac, foi realizado um estudo quase experimental, onde foi elaborado um flip chart do tipo livro sobre anatomia radicular. Por meio de pesquisa bibliográfica, os residentes compilaram informações e imagens sobre o assunto e posteriormente, com o uso de plasticina profissional, criaram modelos anatômicos 3D que foram transformados em imagens digitais por meio de um software; Essas imagens foram então usadas para projetar e criar o flip chart de realidade aumentada. Para avaliar a consolidação do conhecimento, foi utilizada uma avaliação diagnóstica Knwoledge and Prior Study Inventory (KPSI) antes da atividade, e uma avaliação somativa ao final de sua implantação. O teste diagnóstico mostrou que $60 \%$ dos residentes iniciaram a pós-graduação com uma categoria de conhecimento não consolidado. Após a implementação do desenho didático do flipchart de realidade aumentada, elaborado 


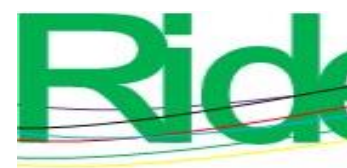

Revista Iberoamericana para la Investigación y el Desarrollo Educativo ISSN 2007 - 7467

pelos alunos com orientação e apoio do professor, observou-se que $90 \%$ dos residentes alcançaram a categoria de conhecimento consolidado em sua avaliação; por fim, aplicou-se o teste estatístico qui quadrado (X2) para verificar se havia relação entre as variáveis analisadas. O resultado do teste mostrou que a implementação do flipchart de realidade aumentada impactou na consolidação do conhecimento da anatomia radicular. Em conclusão, os resultados obtidos mostraram que a incorporação dos novos modelos educacionais baseados em competências e novas tecnologias é favorável para a consolidação de conhecimentos e aprendizagem instrumental e procedimental.

Palavras-chave: aprendizagem, design didático, flip chart, tecnologia educacional, tratamento de canal radicular.

Fecha Recepción: Junio 2020

Fecha Aceptación: Noviembre 2020

\section{Introducción}

La endodoncia — como lo señalan Cohen y Hargreaves (2016) — "es la rama de la odontología encargada de la prevención, diagnóstico, y tratamiento de las enfermedades pulpares y periapicales. Siendo el tratamiento de conductos la terapéutica indicada para preservar los dientes que de otra forma estarían condenados a la extracción” (p. 15). Para un adecuado tratamiento de conductos es indispensable - como lo refieren Ingle y Rotstein (2019) - lograr una buena instrumentación del sistema de conductos radiculares, proceso que consiste en la eliminación por completo de microorganismos y tejido pulpar mediante la ampliación del diámetro y crear una configuración del conducto radicular que permita una obturación y un sellado óptimo.

Sin embargo, una de las principales causas de fracaso del tratamiento de conductos (endodoncia) es el conocimiento inadecuado de la anatomía radicular y sus variaciones. Estos hitos anatómicos, si no se manejan de forma correcta, pueden llevar a errores de procedimiento tales como perforaciones, transportaciones apicales, formación de escalones, pérdida de longitud de trabajo, fractura de instrumentos, entre otros, lo que disminuye el pronóstico del tratamiento y en algunos casos puede producir la pérdida del órgano dentario. Por ello, es de suma relevancia — como lo proponen Cohen y Hargreaves (2016) - que el proceso de enseñanza y aprendizaje de la anatomía radicular y sus variaciones sea eficaz y efectivo para que el residente cuente con el conocimiento adecuado para realizar tratamientos de calidad y así resolver los casos clínicos a los que se enfrenta. 


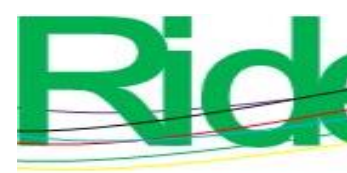

Revista Iberoamericana para la Investigación y el Desarrollo Educativo ISSN $2007-7467$

El problema en la enseñanza y aprendizaje de la anatomía radicular radica en que el alumno no la puede visualizar clínicamente, por lo que la enseñanza se basa en descripciones y esquemas de libros de texto, herramientas que son insuficientes para poder llevar a cabo una construcción adecuada del conocimiento, lo que reduce su estudio a una cuestión meramente memorística que no abona a una correcta correlación con su práctica clínica ni a desarrollar habilidades conceptuales y procedimentales. Todo eso, por el contrario, fomenta la posibilidad de cometer errores de procedimiento que comprometerán el éxito de los tratamientos. A diferencia, por ejemplo, del estudio de anatomía de cráneo, el residente puede realizar ejercicios y prácticas con los huesos de cráneo teniendo así la posibilidad de ver directamente sus características, visualizar sus elementos e interactuar físicamente con sus estructuras. De ese modo se familiariza y comprende mejor las descripciones anatómicas de los textos.

Dado que el aprendizaje de estos conceptos es clave dentro del plan curricular del posgrado, se deben adoptar diseños instruccionales que acompañen al alumno en un ambiente de interés y motivación para que sea partícipe de la construcción del conocimiento. Como menciona Papert (1999), “el mejor aprendizaje no derivará de encontrar mejores formas de instrucción, sino de ofrecer al educando mejores oportunidades para construir" (p. 6). En efecto, cuando se tratan temas que involucran un quehacer práctico, el constructivismo juega un papel crucial. Tomando en cuenta dicho paradigma, Bers, Strawhacker y Vizner (2018) establecen que es posible que el alumno interactúe simultáneamente con dos esferas: la del conocimiento abstracto (información teórica de las características anatómicas) y la de lo sensorial (experiencia física y clínica). Así se le permite al alumno tomar un rol central y mucho más activo en el aprendizaje de contenidos, en comparación con otros métodos que posicionan al estudiante como un mero receptor de información.

En la actualidad, en el área odontológica —y más en específico en la especialidad de endodoncia - hay poca literatura acerca del uso de la realidad aumentada como recurso educativo en el proceso de enseñanza y aprendizaje y su impacto en las habilidades procedimentales y conceptuales de los alumnos. De hecho, son los métodos de enseñanza tradicionales los que imperan en la formación de los nuevos profesionales de la salud bucal, de ahí que surja la oportunidad para evaluar, por medio de un estudio cuasiexperimental, si la implementación de materiales didácticos con las nuevas tecnologías de la información y conocimiento - apoyados con una concepción constructivista - sirven para consolidar 


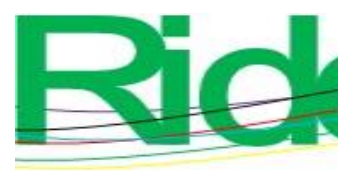

Revista Iberoamericana para la Investigación y el Desarrollo Educativo ISSN 2007-7467

conocimientos en diversas áreas de la odontología (en este caso, la anatomía radicular en la especialidad de endodoncia). La consolidación de conocimientos es aquello que se sabe y no requiere esfuerzo cognitivo para expresarlo o recordarlo; son aquellos conocimientos que se pueden explicar porque se entienden.

La realidad aumentada es una de estas nuevas tecnologías que se pueden usar en el ámbito educativo, ya que permite extender la percepción de la realidad por medio de elementos digitales. Se puede conceptualizar como la combinación de herramientas de un entorno real con otras virtuales que han sido creadas en tres dimensiones. Estos dos aspectos se combinan en tiempo real ampliando la percepción visual de los individuos.

El rotafolio tipo libro, por otra parte, es un medio gráfico elaborado con una serie de hojas de papel unidas en la parte superior, lo que permite ir pasando el contenido; de este modo se mezclan textos e imágenes (gráficas, fotos, dibujos, esquemas) que desarrollan un tema de forma lógica. El proyecto de construcción de este material de realidad aumentada está basado en lo que Papert (1987) considera como “objetos para pensar” (objects-to-thinkwith). Según este autor, nuestro entendimiento del mundo se potencia al crear artefactos, experimentar físicamente con ellos, modificarlos y analizar cómo funcionan.

Explicado lo anterior, se puede decir que el objetivo del estudio consistió en que el residente del posgrado de Endodoncia — por medio de este proyecto de realidad aumentadacreara, manipulara y experimentara con las diferentes formas y variaciones de la anatomía radicular para que lograra construir y consolidar el conocimiento respecto a este tópico. Así se procuró impactar de forma directa en su práctica clínica al lograr mejorar sus habilidades de diagnóstico anatómico y, por ende, realizar tratamientos más predecibles y exitosos.

\section{Materiales y métodos}

Se considera que el estudio es cuasiexperimental debido a la manipulación intencional de la variable independiente (rotafolio de realidad aumentada), además de que se midió la repercusión que con su creación, aplicación e implementación tuvo en la variable dependiente (conocimientos y aprendizajes procedimentales y conceptuales de la anatomía radicular de los residentes de la especialidad); por ello, se establece una relación causa y efecto. Aunado a ello, vale destaca que en este trabajo no se pudieron controlar de manera rigurosa todas las variables involucradas (tales como nivel de conocimientos previos del tema, edad o condición socioeconómica) y tampoco existió un grupo control.

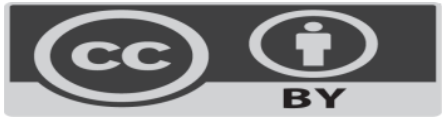




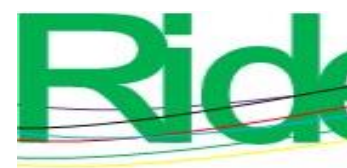

Revista Iberoamericana para la
Investigación y el Desarrollo Educativo ISSN 2007-7467

El estudio se realizó en el posgrado de Endodoncia de la Universidad Tecnológica de México (UNITEC, México), campus Marina-Cuitláhuac, con la participación de 10 residentes de primer año. Para el proyecto se contó con la aprobación del comité de ética y del departamento de investigación de la facultad; además, se consiguió el consentimiento de los alumnos participantes, a quienes se les resguardaron sus datos personales.

Se escogió este grupo de sujetos debido a que ellos ingresan a la especialidad con los aprendizajes acerca de la anatomía radicular adquiridos de forma tradicional en los programas de pregrado. Por eso, se consideró como un grupo idóneo para evaluar el impacto de la implementación de esta estrategia de naturaleza constructivista.

Los participantes fueron hombres y mujeres con edades entre los 24-35 años, con nivel de estudios de licenciatura (60\% de escuelas públicas y $40 \%$ de escuelas particulares), y tenían experiencia laboral (mínimo un año) en el ámbito odontológico. Se caracterizaron por provenir de ambientes urbanos, de clase media y media-alta. En concreto, $50 \%$ provenía de la zona metropolitana del Valle de México, y $50 \%$ del interior de la república.

La ubicación temporal fue el año 2019, en el primer año de la residencia, ya que ese momento es el idóneo para poder evaluar los aprendizajes conceptuales y procedimentales con los que ingresan los residentes a la especialidad. De esa forma se reconocen fallas, carencias y limitaciones en torno al tema anatomía radicular. Así, se pudo contrastar el diagnóstico realizado con los resultados recabados con la implementación de la estrategia didáctica aplicada.

Este proyecto de investigación tuvo tres etapas en su desarrollo: la primera consistió en la explicación a los alumnos del proyecto de investigación. Esto permitió conseguir la libertad en la participación, la aceptación y la firma consensuada de todo el grupo. Posteriormente, en el aula se aplicó un examen diagnóstico de tipo KPSI, para evaluar el conocimiento previo asociado al modelo tradicional de enseñanza de las variantes de la anatomía radicular. A continuación, se implementaron dos estrategias didácticas innovadoras basadas en las tecnologías de la información y comunicación (TIC): flipped class junto con makerspace; con ambas el docente — por medio de recursos digitales - guía las actividades de los residentes de forma asíncrona y a distancia. Además, las dos permiten que los alumnos utilicen recursos tecnológicos para desarrollar su parte creativa por medio del diseño y generación de ideas. Esto con el objetivo de fomentar un trabajo colectivo apoyado en las 


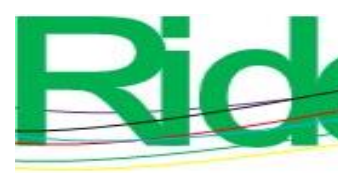
Revista Iberoamericana para la
Investigación y el Desarrollo Educativo
ISSN $2007-7467$

tecnologías educativas, entre ellas el rotafolio con realidad aumentada, el cual fue creado por los alumnos con la guía y orientación del docente.

La tercera etapa fue presencial en el aula. Esta permitió concretar los conocimientos y aprendizajes conceptuales y procedimentales de la anatomía radicular (diagnóstico radiográfico, diagnóstico y características clínicas, sistemas de clasificación, opciones de tratamiento), construidos a lo largo de la actividad a través de la presentación de cada alumno de su parte correspondiente del rotafolio y finalmente aplicando una evaluación de revisión de casos.

\section{Evaluación diagnóstica KPSI}

Esta evaluación fue pensada como un cuestionario de autoevaluación que sirvió para obtener información acerca de la percepción del nivel de conocimientos y de los aprendizajes conceptuales y procedimentales que tenía el alumno respecto a la anatomía radicular, así como las áreas donde podía mejorar. También dio información clave para contrastar al final de la investigación si la implementación de este modelo de aprendizaje consolidó los aprendizajes de esta asignatura. Esto difiere con la evaluación sumativa, ya que se pensó más como un análisis de casos clínicos que brindaría información acerca de cómo el alumno consolidó los conocimientos y los pudo aplicar en su práctica clínica, situación que no se lograría establecer al inicio del curso debido a que aún no se habían presentado y discutido los temas de las variantes anatómicas. Para esta prueba diagnóstica se elaboraron cinco reactivos para cada una de las anomalías a estudiar de la anatomía radicular; las opciones de respuestas a estos cuestionamientos se determinaron en cinco niveles; 0) no lo sé, 1) lo sé un poco, 2) lo sé, pero no en su totalidad, 3) lo sé bien y 4) lo podría explicar a otro colega/profesor.

Las preguntas fueron construidas con el apoyo de tres docentes del posgrado de Endodoncia de la Universidad Tecnológica de México para detectar el nivel de conocimiento de cuatro grandes rubros de la anatomía radicular: diagnóstico clínico, características anatómicas y clasificación, hallazgos radiográficos y, por último, plan de tratamiento. De esta manera se obtuvieron datos que nos permitieron identificar los alcances del modelo educativo tradicional de enseñanza de la anatomía radicular para posteriormente contrastarlos una vez aplicada nuestra propuesta de técnica didáctica constructivista. 


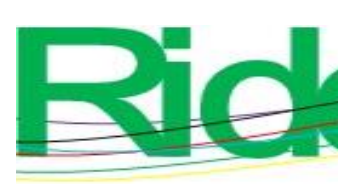

Revista Iberoamericana para la
Investigación y el Desarrollo Educativo ISSN 2007-7467

\section{Flipped classroom (aula inversa)}

Una vez que se llevó a cabo el examen diagnóstico, el docente dio indicaciones a los alumnos para las siguientes fases: en la primera, el alumno ingresó a la plataforma Edmodo para revisar el contenido elaborado y proporcionado por el docente, que consistió en una presentación en Power Point donde se logró conocer y analizar el tema Generalidades de la anatomía radicular, fundamental para que en fases posteriores del trabajo el alumno pudiera ir concretando la información que colocaría en las hojas del rotafolio. En dicha presentación el alumno también tuvo acceso a las instrucciones para hacer la actividad de rotafolio con realidad aumentada, a la vez que pudo consultar el cronograma de actividades.

\section{Makerspace (espacio de creación)}

En la segunda sección de la presentación se encontraron las instrucciones proporcionadas por el docente para el diseño del rotafolio: la primera de ellas consistió en la asignación individual de los diferentes temas de las variantes anatómicas radiculares. Posteriormente, los residentes tuvieron un plazo de dos semanas para recopilar información acerca del tema asignado, enfocándose en el aspecto clínico, hallazgos radiográficos, características clínicas, clasificación y opciones de tratamiento de la anomalía asignada. Además, por medio de las siguientes páginas web (Facebook, Instagram y Youtube) los residentes realizaron búsqueda especializada para identificar, conocer y extraer imágenes, tanto de modelos digitales como reales, de las características morfológicas de su tema.

Luego, los residentes realizaron modelos $3 \mathrm{D}$ con plastilina profesional, en los horarios y lugares que ellos eligieran. Dichos modelos fueron basados en las características anatómicas de cada anomalía, siguiendo las pautas de dimensiones establecidos previamente en la presentación, de manera que se obtuvo al final una réplica de un órgano dental con las características morfológicas de su variante anatómica asignada (figura 1). Durante este proceso el equipo tomó fotografías y videos como evidencia de trabajo y se enviaron a través de la aplicación Whatsapp al docente, quien las resguardó y almacenó en carpetas digitales para retroalimentar o consultar dudas.

Obtenidos los modelos, los alumnos acudieron a un centro de escaneo 3D para obtener imágenes digitales tridimensionales; esos archivos fueron recopilados en memorias USB y llevados a sus equipos de cómputo. El siguiente paso fue usar la aplicación Augment para subir sus imágenes de los diversos modelos y tenerlas listas para la siguiente fase en forma 


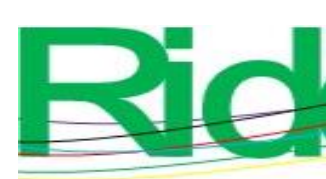

Revista Iberoamericana para la Investigación y el Desarrollo Educativo ISSN 2007-7467

de "rastreadores" (imágenes QR que contienen los modelos 3d de realidad aumentada previamente subidos).

Figura 1. Diseño anatómico terminado (se utilizó pintura acrílica para su acabado)

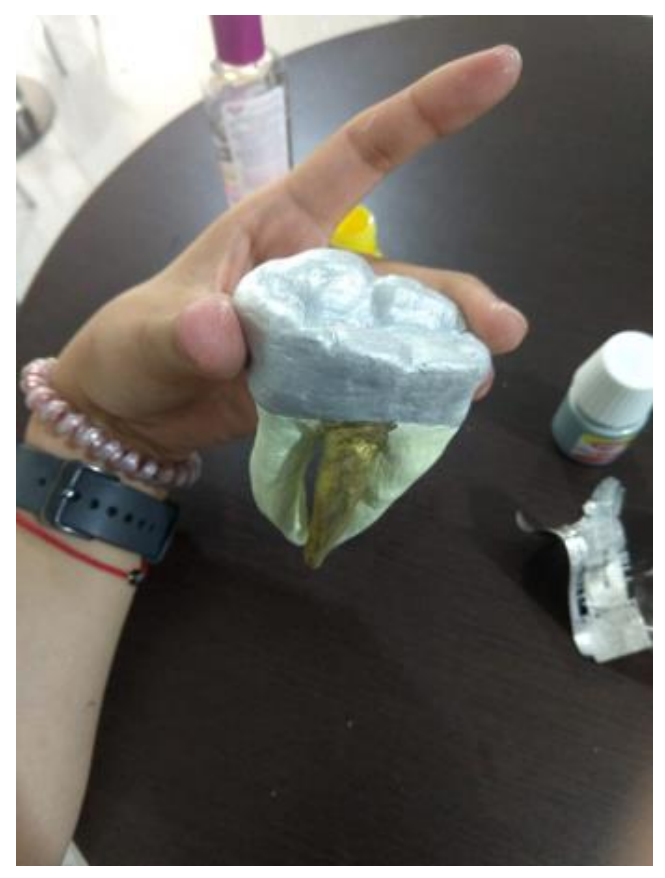

Fuente directa

\section{Elaboración rotafolio de realidad aumentada}

Los residentes - con base en las lecturas, investigación de literatura (guiada por el docente) y presentación del tema proporcionada por el profesor- elaboraron las diferentes hojas del rotafolio tipo libro de las variantes de la anatomía radicular. Cada hoja del rotafolio correspondió a un alumno; el diseño fue a libre criterio, pero con la pauta de cubrir los siguientes rubros en su contenido: aspecto clínico, hallazgos radiográficos, características clínicas, clasificación y opciones de tratamiento. Se tomaron fotos a las diversas páginas y se enviaron al docente por medio de la aplicación Whastapp; el docente revisó el contenido y ofreció retroalimentación, de tal manera que se dio un visto bueno acerca de la información plasmada en la hoja de rotafolio. Después el residente, por medio de la plataforma Augment, añadió los rastreadores, al diseño de su tema, para que al final cada hoja del rotafolio tuviera una imagen $\mathrm{QR}$ que funcionaba como código de almacenaje de los modelos 3D (figura 2). 

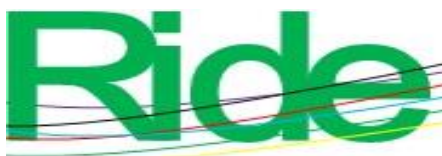

Revista Iberoamericana para la Investigación y el Desarrollo Educativo ISSN 2007 - 7467

Figura 2. Hoja del rotafolio (contiene el desarrollo del tema y el código QR que alberga la imagen anatómica 3D)

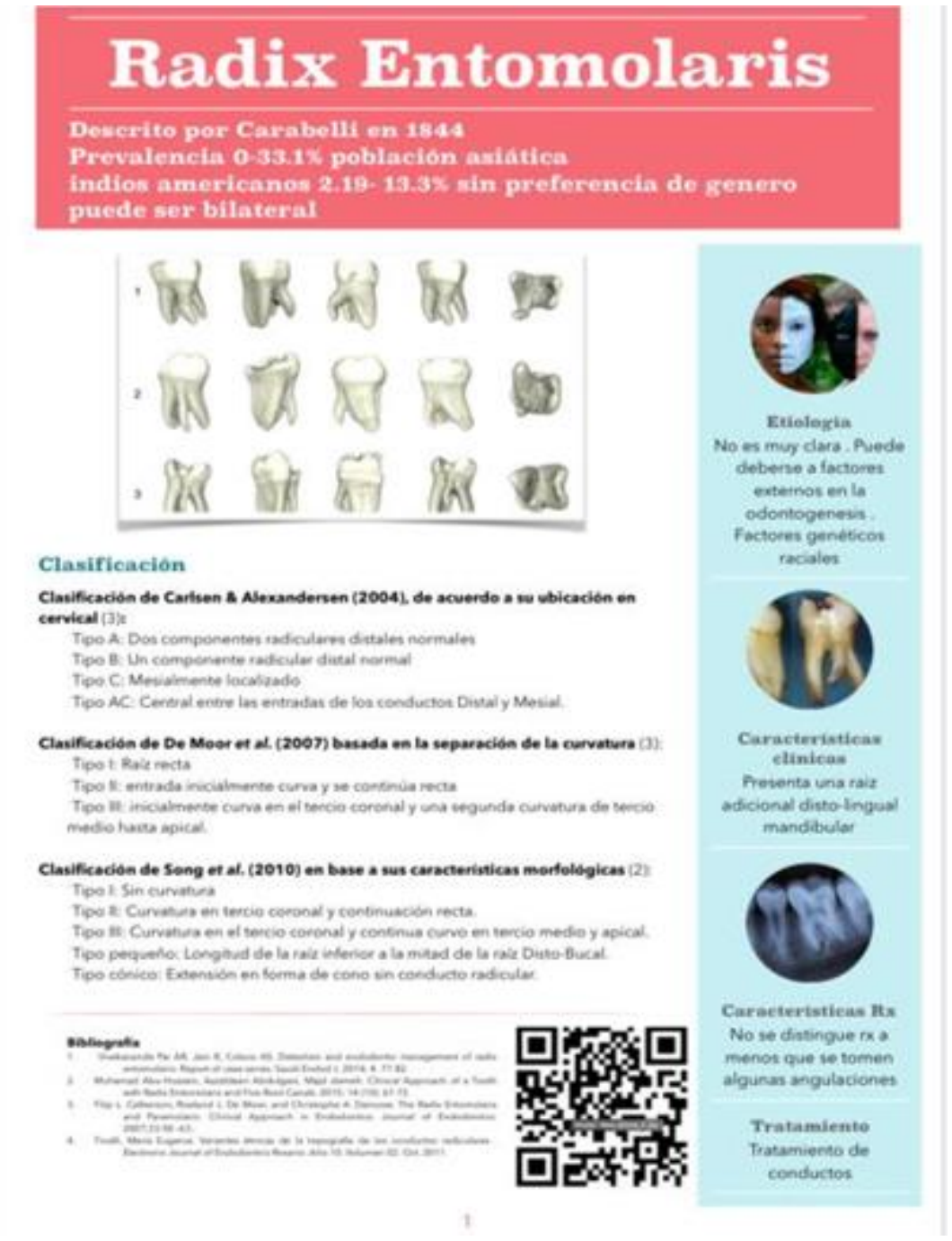

Fuente directa

\section{Presentación de rotafolios}

Al estar listas las diez hojas del rotafolio se procedió a compartirlas, de tal manera que cada alumno construyó su propio rotafolio con todos los tópicos asignados; posteriormente, se organizó una sesión presencial en donde los equipos presentaron los trabajos obtenidos, teniendo como dinámica que los alumnos seguían la exposición del orador revisando los modelos de realidad aumentada de cada tema con sus dispositivos inteligentes, siendo este un espacio en el cual se debatió y se retroalimentó el contenido (figura 3). 


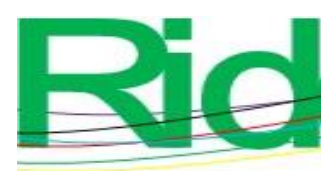

Revista Iberoamericana para la Investigación y el Desarrollo Educativo ISSN 2007-7467

Figura 3. Presentación del rotafolio ( se utilizó el diseño anatómico 3D de cada tema como apoyo y guía de la exposición)

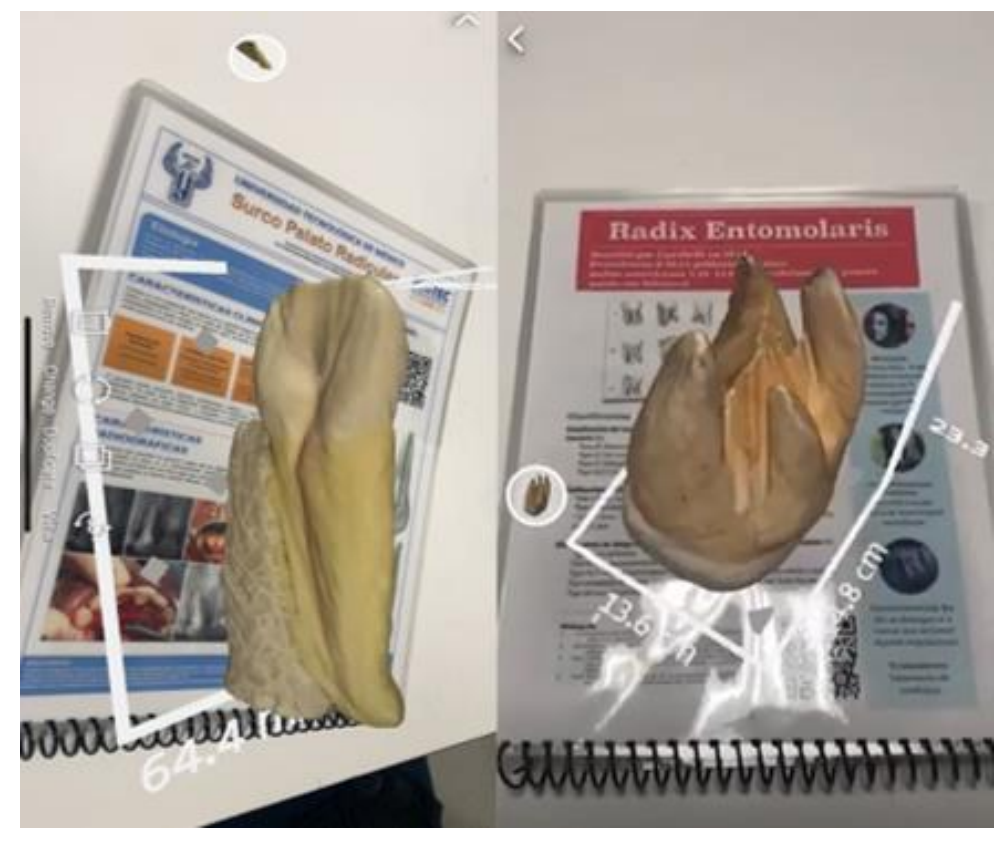

Fuente directa

\section{Evaluación sumativa}

Este segundo instrumento se aplicó al final de la creación e implementación del rotafolio de realidad aumentada; con esta prueba de tipo sumativo se planteaba obtener información acerca del impacto que tuvo el diseño, creación e implementación de esta estrategia didáctica en el conocimiento y los aprendizajes procedimentales y conceptuales de la anatomía radicular que tuvieron los residentes de la especialidad, y así contrastarlos con los datos obtenidos con la prueba diagnóstica KPSI.

Para la creación de la prueba se desarrollaron dos casos clínicos con el apoyo de tres profesores del posgrado de Endodoncia. Se tomaron como base casos reales presentados en la clínica del posgrado, y se tuvo como parámetros el diagnóstico, las características anatómicas, los hallazgos radiográficos y el plan de tratamiento. 


\section{Prueba estadística}

La prueba estadística que se decidió utilizar fue la de chi cuadrado $\left(\mathrm{X}^{2}\right)$ debido a que determina si dos variables están relacionadas o no, por lo cual es adecuada para este trabajo, ya que el estudio pretendió evaluar si la aplicación de una estrategia didáctica de realidad virtual está relacionada con una consolidación de conocimientos acerca de las variantes de la anatomía radicular.

\section{Resultados}

Después de realizar el trabajo de campo se capturaron los datos obtenidos por medio de tablas en el programa Excel del paquete Office 365, las cuales se muestran en la tabla 1 (Prueba KPSI) y tabla 2 (evaluación de casos). El hecho de ser pruebas diferentes al inicio y final del proyecto no afectó el análisis de datos, ya que se pudieron unificar los resultados al categorizarlos en niveles de conocimiento, de esta manera se logró establecer un punto de comparativa para aplicar instrumentos estadísticos. La prueba diagnóstica tuvo que ser diferente debido a que al inicio de la asignatura los residentes aún no habían revisado los tópicos de la asignatura, por lo cual no sería válido establecer una prueba de casos clínicos.

Tabla 1. Resultados de la prueba KPSI

\begin{tabular}{|l|l|l|l|l|l|l|}
\hline Alumno & $\begin{array}{l}\text { Diagnóstico } \\
\text { radiográfico }\end{array}$ & $\begin{array}{l}\text { Diagnóstico } \\
\text { clínico }\end{array}$ & $\begin{array}{l}\text { Características } \\
\text { anatómicas }\end{array}$ & $\begin{array}{l}\text { Plan de } \\
\text { tratamiento }\end{array}$ & $\begin{array}{l}\text { Puntaje } \\
\text { final }\end{array}$ & $\begin{array}{l}\text { Nivel de } \\
\text { conocimiento }\end{array}$ \\
\hline 1 & 34 & 34 & 30 & 32 & 130 & Bueno \\
\hline 2 & 24 & 28 & 26 & 27 & 104 & Suficiente \\
\hline 3 & 25 & 36 & 25 & 29 & 106 & Suficiente \\
\hline 4 & 34 & 36 & 34 & 32 & 136 & Bueno \\
\hline 5 & 25 & 25 & 27 & 24 & 101 & Suficiente \\
\hline 6 & 22 & 22 & 26 & 23 & 93 & Suficiente \\
\hline 7 & 26 & 24 & 24 & 21 & 95 & Suficiente \\
\hline 8 & 28 & 26 & 24 & 25 & 103 & Suficiente \\
\hline 9 & 36 & 36 & 36 & 36 & 144 & Excelente \\
\hline 10 & 31 & 30 & 34 & 29 & 124 & Bueno \\
\hline Total & 285 & 297 & 286 & 278 & 1136 & \\
\hline
\end{tabular}

Nota: (E) Excelente=144 (B) Bueno= 108-143 (S) Suficiente= 72-107 (I) Insuficiente= 071.

Fuente: Elaboración propia 
Tabla 2. Resultados de la prueba sumativa

\begin{tabular}{|c|c|c|c|c|c|c|}
\hline Alumno & $\begin{array}{l}\text { Diagnóstico } \\
\text { radiográfico }\end{array}$ & $\begin{array}{l}\text { Diagnóstico } \\
\text { clínico }\end{array}$ & $\begin{array}{l}\text { Características } \\
\text { anatómicas }\end{array}$ & $\begin{array}{l}\text { Plan de } \\
\text { tratamiento }\end{array}$ & $\begin{array}{l}\text { Puntaje } \\
\text { final }\end{array}$ & \begin{tabular}{|l|l|} 
Nivel de \\
conocimiento
\end{tabular} \\
\hline 1 & 3 & \begin{tabular}{l|l}
2 &
\end{tabular} & 2 & 3 & 10 & Bueno \\
\hline 2 & 3 & 3 & 2 & 3 & 11 & Bueno \\
\hline 3 & 3 & 3 & 3 & 3 & 12 & Excelente \\
\hline 4 & 3 & 3 & 2 & 3 & 11 & Bueno \\
\hline 5 & 3 & 3 & 3 & 3 & 12 & Excelente \\
\hline 6 & 2 & 2 & 2 & 1 & 7 & Suficiente \\
\hline 7 & 2 & 2 & 3 & 3 & 10 & Bueno \\
\hline 8 & 3 & 2 & 2 & 2 & 9 & Bueno \\
\hline 9 & 3 & 3 & 3 & 3 & 12 & Excelente \\
\hline 10 & 3 & 3 & 3 & 3 & 12 & Excelente \\
\hline Total & 28 & 26 & 25 & 27 & 106 & \\
\hline
\end{tabular}

Nota: Excelente=12 Bueno=8-11 Suficiente=4-7 Insuficiente 0-3.

Fuente: Elaboración propia

Para efectos de este estudio se determinó que el alumno tenía una consolidación de conocimientos si alcanzaba el nivel de bueno o excelente en la evaluación diagnóstica; asimismo, se consideró que lograba o mantenía una consolidación después de la aplicación del diseño didáctico cuando obtenía la categoría bueno o excelente, quedando para ambas evaluaciones una categoría de no consolidado con nivel de suficiente o insuficiente.

La prueba KPSI muestra que $60 \%$ de los residentes contaban con un conocimiento de categoría "suficiente" respecto a las variantes anatómicas radiculares, observándose que la mayoría de los alumnos ingresaban a la especialidad con un modelo tradicional de enseñanza que se enmarcaba en el nivel de conocimiento "no consolidado". También sobresale que ninguno de los participantes obtuvo una calificación de "insuficiente" para esta prueba.

Del análisis de estos elementos se observa que el apartado de "Diagnóstico clínico" es el área de conocimiento mejor consolidado (mayor puntaje alcanzado), mientras que el "Plan de tratamiento" consiguió menor puntaje con respecto de los demás.

Por su parte, en la prueba sumativa llevada a cabo después de haber implementado el diseño didáctico del rotafolio de realidad aumentada muestra que $90 \%$ de los participantes alcanzó en sus resultados la categoría de "bueno" a "excelente". Solo un residente obtuvo "suficiente" en su prueba de análisis de casos. 


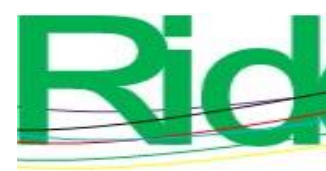

을

Revista Iberoamericana para la Investigación y el Desarrollo Educativo

ISSN 2007 - 7467

El área que tuvo mayor avance de consolidación fue el "Diagnóstico radiográfico", que pasó de ser el penúltimo lugar en la prueba diagnóstica a ser el primero en la sumativa, seguido del apartado de "Plan de tratamiento", que pasó del último lugar en el examen diagnóstico al segundo puesto en la prueba sumativa.

\section{Análisis chi cuadrado}

Posteriormente, usando el programa Excel 360 se realizó el llenado de la tabla de contingencia para el análisis estadístico de chi cuadrado. Con estos datos se procedió a llevar a cabo la prueba; en primera instancia se obtuvieron las frecuencias observadas y frecuencias esperadas, las cuales se observan en la tabla 3 , y después se realizaron los cálculos de la fórmula:

$$
x 2=\frac{\sum(\mathrm{oi}-\mathrm{ei}) 2}{\mathrm{ei}}
$$

En donde $o_{i}$ representa a cada frecuencia observada y $e_{i}$ representa a cada frecuencia esperada. Cabe señalar que el margen de error para este caso se determinó en 0.05 y para el grado de libertad se obtuvo un valor de 1.

Tabla 3. Chi2 correspondiente a cada una de las frecuencias observadas y frecuencias esperadas

\begin{tabular}{|c|c|c|}
\hline $\begin{array}{c}\text { Frecuencia } \\
\text { observada }\end{array}$ & $\begin{array}{c}\text { Frecuencia } \\
\text { esperada }\end{array}$ & Chi 2 \\
\hline 9 & 6.5 & 0.96153846 \\
\hline 4 & 6.5 & 0.96153846 \\
\hline 1 & 3.5 & 1.78571429 \\
\hline 6 & 3.5 & 1.78571429 \\
\hline & & 5.49450549 \\
\hline
\end{tabular}

Fuente: Elaboración propia

Conseguido el valor de chi 2 (el cual fue 5.4945), se contrastó con el valor de Distribución de chi 2 por tabla, que corresponde a 3.8415 para un margen de error de 0.05 y grado de libertad de 1 , obteniendo un valor de $\mathrm{P}<0.05$. 

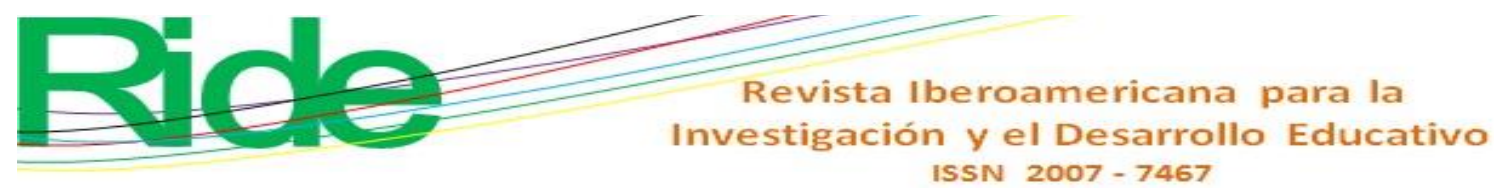

Para la realización del estudio se planteó la utilización del formulario KPSI, el cual está diseñado como una prueba autodiagnóstica de conocimientos. Este cuestionario fue ideal, ya que se pretendía conocer el nivel de conocimiento de la asignatura de anatomía dental con el que los alumnos ingresaban al programa de la especialidad; posteriormente, se contrastaba con una prueba sumativa basada en resolución de casos clínicos, una vez que los alumnos habían repasado los diferentes tópicos de la anatomía radicular de la asignatura (Tamir y Lunetta, 1978).

La idea de incorporar la realidad aumentada como recurso tecnológico de aprendizaje concuerda con lo planteado por Díaz-Barriga y Hernández (2010), quienes mencionan que el aprendizaje para que sea adecuadamente procesado por el alumnado debe ser significativo, marcar un punto de inflexión en el receptor de la información, de tal manera que no pase a ser un dato olvidable o prescindible. Por otra parte, se considera que este diseño es de corte constructivista porque sigue lo planteado por Piaget (1974), quien menciona que desde la perspectiva epistemológica el constructivismo se enfoca en hacer un análisis del conocimiento, estudiando sus limitantes y alcances. En otras palabras, el conocimiento se construye de la interacción que los individuos tienen con su medio ambiente, es decir, con la manipulación de su entorno. De acuerdo con Barlett (referenciado por Good y Brophy, 1990), el constructivismo se sustenta en lo siguiente:

El que aprende construye su propia realidad o al menos la interpreta de acuerdo con la percepción derivada de su propia experiencia, de tal manera que el conocimiento de la persona es una función de sus experiencias previas, estructuras mentales y las creencias que utiliza para interpretar objetos y eventos (p. 55).

Para esta investigación las dimensiones de aprendizaje que se involucraron fueron las de recepción y descubrimiento significativos, según lo documentado por Ausubel (1983). El primero de ellos es el descubrimiento significativo: el alumno a través de la manipulación de materiales crea y descubre las diversas formas anatómicas y sus intrincados elementos estructurales, que de otra manera no se podría debido a las limitaciones clínicas involucradas. De esta manera, la nueva información es significativa en la medida que se relaciona con la ya existente en la estructura cognitiva de forma sustantiva (esquemas de libros, por ejemplo). En un segundo aspecto se considera la recepción significativa, la cual se presenta en el momento en que el rotafolio está listo y se da la fase de presentación del trabajo, escenario 


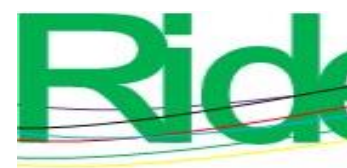

Revista Iberoamericana para la Investigación y el Desarrollo Educativo ISSN $2007-7467$

en donde todos los residentes exponen a sus compañeros el producto final y de esta manera los demás alumnos internalizan el contenido en su estructura cognitiva, diferenciándolo del simple aspecto memorístico.

Por su parte, Fabregat (2012) establece que la creación de diseños didácticos con base en la realidad aumentada beneficia el proceso educativo de varias maneras; por ejemplo, ayuda a la adquisición de conocimientos procedimentales, los cuales son fundamentales, así como para relacionar y comprender los conceptos aprendidos mediante la interacción con los recursos que rodean al individuo.

Sumado a lo anterior, también estamos de acuerdo con Papert (1987), quien menciona que para que el aprendizaje se genere de forma consolidada se debe realizar por medio de la reconstrucción cognitiva y por medio de la práctica. En el presente estudio eso se materializó a través de la manipulación del entorno y sus objetos, es decir, mediante la creación por medio de plastilina de los modelos anatómicos 3D, lo cual se encuentra en sintonía con el concepto objects-to-think (objetos para pensar).

Por otra parte, las tecnologías educativas utilizadas en este trabajo se escogieron con base en lo que Johnson, Becker, Estrada y Freeman (2015), quienes explican que las técnicas flipped classroom y makerspace son algunas de las TIC emergentes con más relevancia debido a sus cualidades y beneficios para el proceso de enseñanza y aprendizaje, de ahí que sean modelos pedagógicos que rápidamente están adquiriendo protagonismo.

De acuerdo con Bers et al. (2018), este tipo de metodologías tiene la ventaja de fomentar en el alumnado un sentido de pertenencia con el contenido que están creando e incrementar la perseverancia al estimular una creación idónea para ellos.

En ese sentido, el presente trabajo coincide con Tekedere y Göker (2016) cuando mencionan que la realidad aumentada tiene un efecto positivo en el aprendizaje de los alumnos; sin embargo, también es conveniente rescatar las palabras de Schmidt y Ralph (2016), quienes advierten que si estas herramientas se usan de forma inadecuada, pueden llevar al conformismo del alumnado y generar un desinterés respecto a la materia, por lo que se sugiere ser cautelosos en este aspecto, ya que el peso que tiene el uso de la realidad aumentada puede estar sobreestimado. Por eso, se debe incluir en trabajos futuros la medición específica del impacto que tiene este tipo de diseños didácticos. 


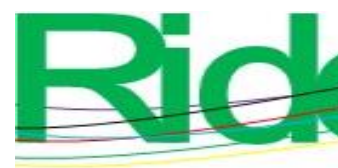

Revista Iberoamericana para la Investigación y el Desarrollo Educativo ISSN 2007-7467

\section{Conclusiones}

La aplicación de la realidad aumentada en nuestra vida cotidiana es cada vez más común, de ahí que los centros educativos se hayan visto en la necesidad de incorporar esta tecnología para fortalecer los procesos de enseñanza y aprendizaje. Áreas como la medicina, enfermería, ingeniería industrial, entre otras, ocupan dispositivos de realidad aumentada para llevar a cabo sus prácticas de laboratorio, con lo cual han tenido excelentes resultados.

El área odontológica, por ende, tiene que incorporar estos nuevos elementos educativos para fortalecer su plan curricular y de esta forma poder ofrecer mejores procesos de enseñanza y aprendizaje a sus alumnos. No obstante, también se debe investigar el área de oportunidad de esta herramienta, pues de ese modo se podrán formar profesionales de la salud bucal mejor preparados en los ámbitos clínicos, éticos y humanistas.

Los modelos educativos basados en el constructivismo, por otra parte, han mostrado ser fundamentales para entender y aplicar los nuevos paradigmas en el sector de la enseñanza. El hecho de dotar al alumno de un papel más activo en el proceso de construcción del conocimiento propicia que se vuelva más crítico, analítico, organizado y con mayor capacidad para resolver problemas a los que se enfrenta, tanto académica como personalmente. Ante esto, el profesorado debe adaptarse a este nuevo rol que le corresponde, en el cual se debe ser un facilitador y guía del proceso, dejando de lado aquella figura dominante cuasipaternal o maternal que ocupaba dentro del proceso educativo.

Este estudio, en síntesis, abre las puertas para repensar el paradigma educativo odontológico actual en México, pues se debe trabajar en la incorporación de nuevas formas de entender los procesos de enseñanza y aprendizaje, donde una tecnología como la realidad aumentada debe ser usada para favorecer la consolidación de conocimientos y habilidades conceptuales y procedimentales de los alumnos.

\section{Agradecimientos}

Un reconocimiento especial a las residentes y los residentes y profesores del posgrado de Endodoncia de la Universidad Tecnológica de México (UNITEC, México), campus Marina-Cuitláhuac que participaron en este proyecto. 


\section{Referencias}

Ausubel, D. P. (1983). Psicología educativa: un punto de vista cognoscitivo (2. ${ }^{\mathrm{a}}$ ed.). Ciudad de México, México: Trillas.

Bers, M. U., Strawhacker, A. and Vizner, M. (2018). The design of early childhood makerspaces to support positive technological development: Two case studies. Library of Hi Tech, 36(1), 75-96.

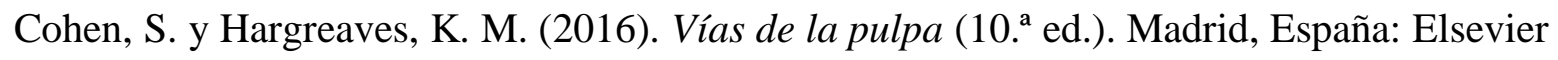
Mosby.

Cubero, P. R. (2005). Perspectivas constructivistas. La intersección entre el significado, la interacción y el discurso. Barcelona, España: GRAO.

Díaz-Barriga, A. F. y Hernández, R. G. (2010). Estrategias docentes para un aprendizaje significativo. Recuperado de http://eoepsabi.educa.aragon.es/descargas/H_Recursos/h_1_Psicol_Educacion/h_1.3 .Aprender_a_aprender/1.11.Estrat_aprendizaje_significativo.pdf

Fabregat, G. R. (2012). Combinando la realidad aumentada con las plataformas de eelearning adaptativas. Enl@ce Revista Venezolana de Información, Tecnología y Conocimiento, 9(2), 69-78.

Good, L. T. and Brophy, J. E. (1990). Educational psychology: A realistic approach (4 ${ }^{\text {th }}$ ed.). Nueva York, Estados Unidos: Longman.

Ingle, I. and Rotstein, I. (2019). Ingle's endodontics ( $7^{\text {th }}$ ed.) North Carolinem, Estados Unidos:BC PMPH.

Johnson, L., Becker S. A., Estrada, V. and Freeman, A. (2015). NMC Horizon Report: 2015 Library Edition. Austin,Texas: The New Media Consortium.

Papert, S. A. (1987). Desafío de la mente. Buenos Aires, Argentina: Ediciones Galápago.

Papert, S. A. (1999). Logo Philosophy and Implementation. Retrieved from https://www.ecoo.org/wp-content/uploads/2018/06/What-is-Logo-And-Who-NeedsIt.pdf

Peñalosa, E. C. (2013). Estrategias docentes con tecnologías: guía práctica. Ciudad de México, México: Editorial Pearson.

Piaget, I. J. (1974). A dónde va la educación. Barcelona, España: Ariel.

Schmidt, S. M. P. and Ralph, D. (2016). The flipped classroom: a twist on teaching. Contemporary Issues in Education Research (Online), 9(1), 16. 


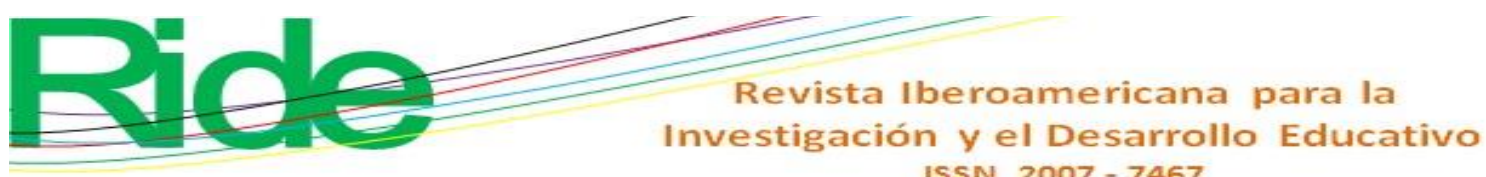

Tamir, P. and Lunetta, V. N. (1978). An analysis of laboratory activities in the BSCS. The American Biology Teacher, 40, 426-428. Doi: 10.2307/4446267

Tekedere, H. and Göker, H. (2016). Examining the effectiveness of augmented reality applications in education: A meta-analysis. International Journal of Environmental and Science Education, 11(16), 9469-9481. 